\title{
TECNOLOGÍAS EN INFORMACIÓN Y COMUNICACIÓN SANITARIA
}

\author{
Miriam Elena Buitrón Vega' ${ }^{1}$ Enrique Gea-Izquierdo 1,2, \\ María Verónica García-Oquendo 3
}

\section{RESUMEN}

La cibersalud, conocida como eSalud, consiste en el uso de las tecnologías de la información y la comunicación en la salud, y otras áreas como la educación y la investigación. La salud es uno de los sectores en los que el uso de las TIC ha penetrado en forma intensiva, usándose en casi todos los ámbitos desde las políticas, la planificación, la información, la investigación, la promoción, prevención, diagnóstico, hasta el tratamiento y rehabilitación de la salud. La confluencia de la ciencia y tecnología, junto con otras prestaciones, transforman la eSalud en un sistema complejo con orientación hacia la salud integral, más allá de la interconexión entre sus componentes. Hay avances sobre la aplicación de las TIC en la salud en el Ecuador, pero pese a las po- tencialidades para mejorar el acceso y calidad de los servicios, estas no han sido completamente explotadas o implementadas en el país. El uso de las TIC en salud enfrenta un complejo marco legal; la autoridad y actores que intervienen en el proceso regulatorio son múltiples, lo cual implica ventajas y limitaciones para su utilización y desarrollo.

Palabras clave: eSalud, tecnologías de la información y comunicación, marco legal.

\section{ABSTRACT}

E-Health is the use of information and communications technology in health and other areas such as education and research. Health is one of the sectors in which the use of ICT has penetrated

\footnotetext{
1 Pontificia Universidad Católica del Ecuador, Facultad de Medicina, Quito, Ecuador (mebuitron@gmail.com). 'Pontificia Universidad Católica del Ecuador, Facultad de Medicina, Quito, Ecuador.

2Universidad de Málaga, Cátedra de Seguridad y Salud en el Trabajo, Málaga, España (enriquegea@yahoo.es).

${ }_{3}^{3}$ Pontificia Universidad Católica del Ecuador, Facultad de Psicología, Quito, Ecuador (mvgarcia@puce.edu.ec).
} 
intensively. It is said to be found in almost all areas from policy, planning, information, research, advocacy, prevention, diagnosis to treatment and rehabilitation of health. In health, the convergence of science and technology with other advances, make e-health in a complex field whose challenge is the provision of comprehensive health and not merely the possibility of interconnecting their components. There is progress in implementing health in Ecuador, but despite the potential of ICT to improve access and quality of health services, these have not been fully exploited or implemented in the country. The use of ICT in health faces a complex legal framework, authority and actors involved in the regulatory process are multiple, which implies advantages and limitations for application and development.

Key words: eHealth, information and communication technologies, legal framework.

\section{INTRODUCCIÓN}

Hasta principios del siglo XX la práctica médica se realizaba con pocos recursos, que consistían principalmente en la formación profesional como médico o doctor y su maletín. Generalmente, la casa, la habitación del enfermo era el lugar utilizado para el diagnóstico, tratamiento y recuperación del paciente con el apoyo de familiares y vecinos; los hospitales eran destinados a aquellos enfermos de escasos recursos económicos.

La medicina evolucionó significativamente, concomitantemente al desarrollo de la física, la fisiología, la ingeniería, la microbiología, etc. que fueron aplicadas al diagnóstico y tratamiento de las enfermedades. El descubrimiento de los Rayos X de W.K. Roentgen, en 1895; el electrocardiograma por Willem Einthoven, en 1903; y el desarrollo de los hos- pitales que dejaron de ser receptores de pacientes pobres y pasaron a ser instituciones para todos los miembros de la sociedad fueron innovaciones significativas para la medicina en el siglo pasado.

En las últimas décadas la innovación tecnológica ha cambiado rápidamente cada una de las facetas de nuestra forma de vida, modificando las relaciones personales que se trasladan a un ambiente no presencial o virtual (Torres Velandia \& García Ponce de León, 2008). Además, hay una preocupación creciente por diversas instancias en el uso de las TIC en salud (Ramos, 2006) y por ende en sus implicaciones en aspectos políticos y sociales. Este trabajo es una aproximación a las aplicaciones, los beneficios y el marco normativo que rige en el Ecuador. 


\section{MÉTODO}

El trabajo es de carácter descriptivo, en donde se realizó un estudio documental con énfasis en la aplicación de las TIC en Salud y un análisis del marco legal vigente en el Ecuador, así como de ciertos organismos internacionales y regionales como Organización Panamericana de la Salud y Comunidad Andina de Naciones.
Se identificaron las principales normas vigentes referentes a salud, telecomunicaciones y TIC, y se analizaron las reglamentaciones fundamentales. Se examinaron contenidos de textos político-normativos de los principales documentos legislativos, como la Constitución (2008), Plan Nacional para el Buen Vivir (PNBV), leyes, decretos y acuerdos.

\section{EL SECTOR SALUD Y LAS NUEVAS TECNOLOGÍAS}

Según la Organización Mundial de la Salud (OMS), la cibersalud, conocida como eSalud', consiste en el uso eficaz y seguro de las tecnologías de la información y la comunicación en la salud y otros ámbitos como los servicios médicos, la vigilancia, el registro sanitario, la educación y la investigación en materia de salud. (Organización Panamericana de la Salud/Organización Mundial de la Salud, 2011) Su evolución ha permitido el desarrollo de otras ramas como son la salud móvil (mHealth) que mediante el uso de dispositivos inalámbricos (teléfonos móviles, equipos de monitorización de pacientes, asistentes personales digitales y otros aparatos) apoyan la práctica de la medicina, la decisión clínica y la salud pública.

Eysenbach (2001) define a ehealth como un campo emergente resultante de la unión de la informática médica, la salud pública y los negocios. La eSalud involucra la interacción del software, hardware, telecomunicaciones e internet. Por una parte el uso de las TIC sirve para apoyar y habilitar servicios de atención médica y otras acciones de salud, mientras que por otra la interoperabilidad proporciona la capacidad a los sistemas y a las organizaciones para comunicarse, intercambiar datos, compartir e integrar información, conocimiento y procesos de manera fácil, segura y fluida, comúnmente haciendo uso de estándares y prácticas de trabajo comunes y compatibles.

Sin duda las TIC permiten ordenar los procesos y flujos de información, la comunicación y coordinación entre cen-

${ }^{1} \mathrm{O}$ eHealth en su terminología en inglés. 
tros prestadores, asegurando información oportuna y de calidad para la atención de salud. También permite reflexionar sobre la forma en que se entrega la atención en salud, el modo en que los profesionales y las instituciones se relacionan con los pacientes. Por tanto, los beneficios de estas nuevas tecnologías, tanto en la medicina como en la salud pública, son múltiples. De hecho, facilita la accesibilidad de los pacientes al sistema de salud, eliminando barreras geográficas, orientando que la prestación de servicios a través de telemedicina no requiera el traslado de los pacientes a los centros sanitarios. La implantación de las nuevas tecnologías de telemedicina podría ser una estrategia para mejorar el modelo actual de prestación de servicios de salud, sustituyendo la lógica de tratar enfermedades por la atención integral cuyo interés es el paciente, planteamiento tanto más necesario cuanto que la población envejece y el número de personas que requieren atención crónica va en aumento. (Rabanales Sotos, Párraga Martínez, Lopez-Torres, Prétel \& Navarro Bravo, 2011) Además, permiten obtener una amplia información de una manera rápida y sencilla mejorando la eficacia y racionalizando el gasto del sistema de salud.

\section{APLICACIÓN DE LAS TIC EN SALUD}

La aplicación de las TIC se realiza en un amplio rango de aspectos que afectan el cuidado de la salud, relacionada no solamente con la atención médica que requieren las personas en forma individual sino incluyendo los servicios de salud, la salud pública y la formación de profesionales y los pacientes. En la prevención, diagnóstico, tratamiento y rehabilitación de pacientes se podría decir que las TIC son aplicables en todos los campos médicos; esto ha dado lugar el desarrollo de la telemedicina que, actualmente, incluye distintos servicios: cirugía, teleradiología, teleconsultas, telefotografía y diagnóstico remoto por imagenología digital térmica.
(Sainz de Abajo, Rodrigues, García Salcines, Burón Fernandez, López Coronado \& de Castro Lozano, 2011) La telemedicina es una herramienta que puede servir para eliminar las barreras geográficas, sociales o culturales. Su aplicación permite proveer atención sanitaria sin importar dónde se encuentre el paciente y el profesional de la salud. (Avella Martínez \& Parra Ruiz, 2013)

Teniendo en cuenta el papel fundamental de las TIC en la salud, la convergencia de distintos elementos como la ciencia y tecnología, la informática, las telecomunicaciones y servicios de alto valor agregado como el internet convier- 
ten a la eSalud en un campo complejo cuyo reto es la provisión de salud integral y no solamente la posibilidad de interconectar los componentes del sistema de salud. De hecho hay que considerar no solo las necesidades de infraestructura tecnológica sobre la que debe funcionar sino la interoperabilidad que permite el intercambio de datos y formas de pensar diferentes. (Carnicero \& Fernández, 2012)

Además, la salud es un tema sensible, dependiendo de las características del sistema de cada país la aplicación de las TIC tendrá un impacto mayor o menor en cada uno de sus componentes. Entendemos como sistema de salud a las instituciones, las personas y los recursos implicados en la prestación de atención de salud a todos los individuos. (Organización Mundial de la Salud, 2003)

Por lo tanto, para la aplicación de las TIC hay que tener en cuenta factores como: el hecho que el sector salud tiene un alto grado de regulación por parte del gobierno o los profesionales de la salud; altamente fragmentado, coexisten los servicios públicos, privados, tradicionales o alternativos donde participan muchos actores y culturas diversas; la gestión es fundamentalmente pública; y el financiamiento es a través de impuestos $u$ otros mecanismos, agentes financieros, quienes recaudan fondos y los asignan a proveedores o compran servicios a nivel nacional y otros niveles que financian directa e indirectamente.

\section{LAS TIC EN EL SECTOR SANITARIO DE ECUADOR}

Tal como ocurre en muchos países el sistema de salud ecuatoriano está fragmentado y segmentado. La prestación de servicios se da principalmente por el subsector público conformado por el Ministerio de Salud Pública (MSP) y las instituciones de la seguridad social: Instituto Ecuatoriano de Seguridad Social (IESS), Instituto de Seguridad Social de las Fuerzas Armadas (ISSFA) e Instituto de Seguridad Social de la Policía Nacional (ISSPOL) para trabajadores, militares y policías respectivamente. Adicionalmente, participan los servicios de salud de las municipalidades e instituciones públicas como prefecturas y otros ministerios.

El sector privado con fines de lucro lo constituyen profesionales de la salud que actúan individual o colectivamente en corporaciones que prestan servicios a quienes pueden pagar; además, está el sector privado sin fines de lucro en las que se encuentran organizaciones de la sociedad civil y de servicio social que actúan independientemente. 
El sistema de salud tiene múltiples financiadores, el MSP se financia por rentas del Estado; el sistema de seguridad social por las cotizaciones de los trabajadores, de los empleadores y el Estado; los sistemas privados a través de aseguradoras o pago directo de aquellas personas con capacidad de pago, u Organizaciones no Gubernamentales (ONG) para los más pobres. Al MSP le corresponde el ejercicio de la rectoría, así como la responsabilidad de la aplicación, control y vigilancia del cumplimiento de la Ley Orgánica del Sistema Nacional de Salud (LOSNS) y las normas dictadas para su vigencia.

En el país hay esfuerzos por incorporar a las TIC. En los últimos años las políticas han mejorado ostensiblemente respecto el acceso a internet; en diversas instancias encargadas de la salud a nivel institucional hay acciones para incorporar a las TIC, pero las cosas no son tan simples. Respecto a la telemedicina, también conocida como telesalud existen proyectos pilotos en zonas alejadas, considerando que pese a que la tecnología para la telemedicina puede ir desde el uso de la simple red telefónica hasta los enlaces satelitales, pasando por el intercambio de señales de video y las teleconferencias remotas para trabajo en grupos; su utilización no se extiende a todo el país. (Universidad Técnica Particular de Loja, 2012).
El desarrollo de la comunicación virtual ha permitido la formación del personal sanitario en distintas modalidades y reflexionar en lo relativo a la forma en que se entrega la salud, ideando otros mecanismos de interacción con los pacientes. También informar a la ciudadanía sobre temas de salud y prevención haciendo uso de webcast y podcast. No cabe duda de que las TIC pueden revolucionar la atención de salud a través de proyectos que fusionen la tecnología Near Field Communication (NFC) y la ingeniería informática (Barrera-Jaramillo \& Gea-Izquierdo, 2013).

Por otro lado, respecto a la planificación, organización, gestión de los servicios y sistemas de salud a través de la información por internet y tecnologías relacionadas existen aplicaciones tan diversas como la historia clínica electrónica, la gestión farmacológica, los sistemas de información y gestión administrativa, la gestión de los recursos requeridos así como también los sistemas para la vigilancia de salud pública. Hasta el momento su uso es limitado, aunque hay esfuerzos por implementar la historia clínica electrónica en todo el país. El MSP ha integrado el Sisalud (Solución Informática para la Gestión Integral de Salud) en 116 centros de salud desde septiembre del 2013 y se prevé que 151 centros estatales contarán con el sistema en el 2016. (Vance, 2015). 
En un sistema de salud, la definición de políticas y programas a nivel nacional e internacional y la toma de decisiones, debe basarse en información que posibilite definir necesidades y los efectos de las políticas. Así mismo, se necesita establecer indicadores que permitan medir y garantizar la salud de calidad, acceso universal, continuidad asistencial y seguridad de los pacientes. (Indarte \& Pazos Gutiérrez, 2011) Si bien existen varias iniciativas a nivel nacional que se orientan hacia el gobierno en línea, al menos por el momento esto no es completo, por ejemplo los grandes prestadores del sistema el MSP y el IESS mantienen autonomía en la información y gestión de servicios.

Pese a las potencialidades de las TIC para mejorar el acceso y calidad de los servicios de salud, estas no han sido completamente explotadas o implementadas en el país, a pesar de que como lo vemos en el siguiente acápite, hay un marco legal extenso y favorable para su aplicación y desarrollo en salud.

\section{MARCO LEGAL \\ PARA LA APLICACIÓN DE LAS TIC EN EL ECUADOR}

El Estado nacional establece el marco legal y también los mecanismos de gestión y/o control necesarios para el funcionamiento de las TIC en el sector salud. Cobra especial importancia la función del MSP, sus respectivas dependencias, institutos y entidades descentralizadas así como determinados actores dentro del marco de la informática, la comunicación y la interoperabilidad entre otros.

En el ámbito internacional de la salud, la Organización Panamericana de la Salud (OPS) estableció la Estrategia y Plan de Acción sobre eSalud, siendo el Ecuador país signatario debería tomar en consideración los criterios orientadores para una política de salud y TIC. El propósito del plan es contribuir al desarrollo sostenible de los sistemas de salud de los Estados miembros. Con su adopción se busca mejorar el acceso y la calidad, proponiendo cuatro acciones estratégicas: garantizar y promover la formulación, ejecución y evaluación de políticas de información y públicas eficaces, integradas y sostenibles sobre el uso e implementación de las tecnologías de las comunicaciones en el ámbito sanitario; mejorar la salud pública a través del uso de herramientas y metodologías; fomentar y facilitar la colaboración horizontal entre los países para el desarrollo de una Agenda Digital en Salud para la región; y gestión del conocimiento, formación en alfabetización digital y tecnologías de la información y la comunicación. (Organización Panamericana de la Salud/Organización Mundial de la Salud, 2011) 
En el ámbito regional, la Comunidad Andina referente a las TIC es la que establece las normas que regulan el proceso de integración y liberalización del comercio de servicios de telecomunicaciones, mediante las cuales se fomenta la flexibilidad del mismo, a fin de alcanzar la creación de un Mercado Común Andino de Servicios, contribuyendo así a la inclusión de la Subregión Andina. (Decisión 462, 1999) Asimismo, desarrolló el Manual para la Producción de Estadísticas de Tecnologías de la Información y la Comunicación de la Comunidad Andina. (Resolución 1415, 2011)

Desde el 2002, la agenda nacional de conectividad es la política de Estado que "constituye una síntesis de los elementos políticos, ideológicos y tecnológicos, globales y locales para mejorar el acceso al uso de las tecnologías de la información para el fortalecimiento de la democracia y el buen gobierno, la promoción de los derechos humanos, [...] el desarrollo de la salud y educación, la promoción de la igualdad de género y la promoción de la diversidad cultural, ... " (Jurado, 2006), favoreciendo la aplica- ción de las TIC en el país. No obstante, la institucionalización de una estrategia transversal como es el caso de la Sociedad de la Información, es un proceso de gran complejidad. Tratándose de un tema nuevo, sus efectos están sobre un amplio espectro en diferentes autoridades del sector público, ciencia y tecnología, autoridades sectoriales para negocios-e, gobierno-e, formación-e y eSalud. (Hilbert, Bustos \& Ferraz, 2005)

Las autoridades y actores que intervienen en el proceso regulatorio nacional en materia de TIC está compuesto fundamentalmente por las siguientes normas:

1. La Constitución del Ecuador 2008 instituye el Sumak Kawsay o Buen Vivir como principio ético, que introduce cambios fundamentales en el país. Esta Constitución instaura un nuevo marco referencial para el manejo conceptual e institucional de la salud así como para las TIC y otros ámbitos relacionados, según se contempla en la tabla 1. 
Tabla 1. Marco Constitucional: Salud, TICs y otros ámbitos relacionados

\begin{tabular}{|c|c|c|c|}
\hline SALUD & TICS & $\begin{array}{l}\text { PROPIEDAD DE LOS } \\
\text { DATOS PERSONALES } \\
\text { Y SEGURIDAD }\end{array}$ & $\begin{array}{l}\text { CIENCIA Y } \\
\text { TECNOLOGÍA }\end{array}$ \\
\hline $\begin{array}{l}\text { - El artículo } 32 \text { estable- } \\
\text { ce que la salud es un } \\
\text { derecho que garantiza } \\
\text { el Estado, cuya realiza- } \\
\text { ción se vincula al ejerci- } \\
\text { cio de otros derechos, } \\
\text { entre ellos al de agua, } \\
\text { alimentación, educa- } \\
\text { ción, cultura física, tra- } \\
\text { bajo, seguridad social, } \\
\text { ambientes sanosy otros } \\
\text { que sustentan el buen } \\
\text { vivir. El Estado garan- } \\
\text { tizará este derecho } \\
\text { mediante políticas } \\
\text { económicas, sociales, } \\
\text { culturales, educativas y } \\
\text { ambientales. }\end{array}$ & $\begin{array}{l}\text { - Elartículo } 16 \text { establece } \\
\text { que todas las personas } \\
\text { en forma individual o } \\
\text { colectiva tienen dere- } \\
\text { cho a: acceso universal a } \\
\text { las TIC; la creación de } \\
\text { medios de comunica- } \\
\text { ción social; acceso en } \\
\text { igualdad de condicio- } \\
\text { nes al uso de las fre- } \\
\text { cuencias del espectro } \\
\text { radiolectrónico para la } \\
\text { gestión de estaciones } \\
\text { de radio y televisión pú- } \\
\text { blicas, privadas y comu- } \\
\text { nitarias, y a bandas libres } \\
\text { para la explotación de } \\
\text { redes inalámbricas. Asi- } \\
\text { mismo, el artículo } 261 \\
\text { expone que el Estado } \\
\text { central tendrá compe- } \\
\text { tencias exclusivas sobre } \\
\text { [...] el espectro radioe- } \\
\text { léctrico y el régimen ge- } \\
\text { neral de comunicacio- } \\
\text { nes y telecomunicacio- } \\
\text { nes. } \\
\text { - Superintendencia de } \\
\text { Telecomunicaciones, ar- } \\
\text { tículo } 213 \text {, es el orga- } \\
\text { nismo técnico de vigi- } \\
\text { lancia, auditoría, inter- } \\
\text { vención y control de las } \\
\text { actividades económicas, } \\
\text { sociales y ambientalesy }\end{array}$ & $\begin{array}{l}\text { - El artículo } 92 \text { estipula } \\
\text { que toda persona, por } \\
\text { sus propios derechos o } \\
\text { como representante le- } \\
\text { gitimado para el efecto, } \\
\text { tendrá derecho a cono- } \\
\text { cer de la existencia y a } \\
\text { acceder a los documen- } \\
\text { tos, datos genéticos, } \\
\text { bancos o archivos de } \\
\text { datos personales e in- } \\
\text { formes que sobre sí } \\
\text { misma, o sobre sus } \\
\text { bienes, consten en enti- } \\
\text { dades públicas o priva- } \\
\text { das, en soporte material } \\
\text { o electrónico. Así mis- } \\
\text { mo, tendrá derecho a } \\
\text { conocer el uso que se } \\
\text { haga de ellos, su finali- } \\
\text { dad, el origen y destino } \\
\text { de información perso- } \\
\text { nal. }\end{array}$ & $\begin{array}{l}\text { - El artículo } 385 \text { men- } \\
\text { ciona que el sistema na- } \\
\text { cional de ciencia, tecno- } \\
\text { logía, innovación y sa- } \\
\text { beres ancestrales, en el } \\
\text { marco del respeto al } \\
\text { ambiente, la naturaleza, } \\
\text { la vida, las culturas y la } \\
\text { soberanía, tendrá como } \\
\text { finalidad: desarrollar } \\
\text { tecnologías e innova- } \\
\text { ciones que impulsen la } \\
\text { producción nacional, } \\
\text { eleven la eficiencia y } \\
\text { productividad, mejoren } \\
\text { la calidad de vida y con- } \\
\text { tribuyan a la realización } \\
\text { del buen vivir. }\end{array}$ \\
\hline
\end{tabular}

Fuente: Constitución, 2008 
2. Plan Nacional para el Buen Vivir, la mayor jerarquía de la norma constitucional se completa en este caso con la herramienta normativa de planificación. El PNBV 20132017, cuyo objetivo 11 se refiere a "asegurar la soberanía y la eficiencia de los sectores estratégicos para la transformación industrial y tecnológica", concreta estos lineamientos obligatorios de política pública dentro del Estado. El objetivo 11.3 específicamente señala la necesidad de democratizar la prestación de servicios públicos de telecomunicaciones y de TIC, [...] y profundizar su uso y acceso universal. (Secretaría Nacional de Planificación y Desarrollo, 2013)

3. Ley del Sistema Nacional de Registro de Datos Públicos, artículo 1, tiene como objeto garantizar la seguridad jurídica, organizar, regular, sistematizar e interconectar la información, así como la eficacia y eficiencia de su manejo, su publicidad, transparencia, acceso e implementación de nuevas tecnologías.

a. Respecto a la accesibilidad y confidencialidad, el artículo 6 establece que son confidenciales los datos de carácter personal, tales como: ideología, afiliación política o sindical, etnia, estado de salud... b. El artículo 12 se refiere a los medios tecnológicos: el Estado, a través del ministerio sectorial con competencia en las telecomunicaciones y en la sociedad de la información, definirá las políticas y principios para la organización y coordinación de las acciones de intercambio de información y de bases de datos entre los organismos e instancias de registro de datos públicos... (Ley del Sistema Nacional de Registro de Datos Públicos, 2010)

4. Ley de Comercio Electrónico y Mensajes de Datos: provee instrumentos jurídicos que permiten el uso de medios electrónicos de intercambio tales como la firma electrónica, a fin de promover el uso de las TIC para el desarrollo del comercio y la provisión de servicios de administración pública. (Ley n. ${ }^{\circ}$ 2002-67, 2002)

5. Ley Especial de Telecomunicaciones Reformada: tiene por objeto normar en el territorio nacional la instalación, operación, utilización y desarrollo de toda transmisión, emisión o recepción de signos, señales, imágenes, sonidos e información de cualquier naturaleza por hilo, radioelectricidad, medios ópticos u otros sistemas electro- 
magnéticos. (Ley Especial de Telecomunicaciones Reformada, 1992)

6. Reglamento General a la Ley Especial de Telecomunicaciones Reformada: tiene como finalidad esta blecer las normas y procedimientos generales aplicables a las funciones de planificación, regulación, gestión y control de la prestación de servicios de telecomunicaciones... el internet se encuentra catalogado como servicio de valor agregado. (Decreto Ejecutivo 1790, 2001)

7. Ley Orgánica de Salud: establece la responsabilidad del MSP, mediante el artículo 6, de participar con el organismo competente, en la investigación y el desarrollo de la ciencia o tecnología en salud, salvaguardando la vigencia de los derechos humanos y respetando los principios bioéticos. (Ley n. ${ }^{\circ} 67$, 2006)

8. LOSNS: en el artículo 29 establece que el MSP, con el apoyo del Consejo Nacional de Salud y la participación de la FUNDACYT, ${ }^{2}$ impulsará una política de investigación orientada a las prioridades nacionales y al desarrollo y transferencia de tecnologías [...]. Evaluará y racionalizará el uso de los recursos tecnológicos para su optimización nacional y promoverá el intercambio científico y tecnológico entre las instituciones del sector. (Ley n. ${ }^{\circ}$ 2002-80, 2002)

9. El Ministerio de Telecomunicaciones y de la Sociedad de la Información, artículo 2 de su creación, es responsable de formular, dirigir, coordinar y evaluar las políticas, planes y proyectos para la promoción de la Sociedad de la Información y del Conocimiento y las Tecnologías de la Información y Comunicación. (Decreto Ejecutivo 8, 2009)

a. El Consejo Nacional de Telecomunicaciones (CONATEL) como ente de administración y regulación de las telecomunicaciones es el organismo que se encarga de la regulación, administración y creación de políticas, [...] en el Ecuador. Además, promueve el uso de las TIC para garantizar el acceso de todos los ecuatorianos a la Sociedad de la Información y fomenta el acceso y uso de internet, así como sus aplicaciones en el ámbito social como educación y salud. (Decreto Ejecutivo 8, 2009)

i. Reglamento a la prestación de servicios de valor agregado (internet): establece las nor-

${ }^{2}$ Actual SENESCYT 
mas y procedimientos aplicables a la prestación de servicios de valor agregado así como los deberes y derechos de los prestadores de servicios de sus usuarios. (Reglamento a la prestación de servicios de valor agregado, 2002)

10. El Ministerio de Salud Pública como Autoridad Sanitaria Nacional, es la entidad encargada de establecer las políticas, normar, regular y vigilar la salud de la población ecuatoriana.

a. El Manual de la Gestión Organizacional por Procesos establece la rectoría, regulación, planificación, coordinación, control y gestión de la Salud Pública ecuatoriana a través de la gobernanza, [...] y desarrollo de la ciencia y tecnología y la articulación de los actores del sistema, con el fin de garantizar el derecho a la salud.

i. El objetivo 8: incrementar el desarrollo de la ciencia y la tecnología según las prioridades sanitarias de la salud.

ii. El artículo 20, expone que la Gestión Estratégica tiene co mo misión: dirigir, implementar, administrar y evaluar la gestión de procesos, tecnolo- gías de la información y comunicaciones [...]

iii. La Dirección Nacional de Tecnologías de la Información y Comunicaciones tiene como misión proponer, implementary administrar políticas, normas y procedimientos que optimicen la gestión y administración de las TIC, [...] así como el soporte tecnológico institucional. (Ministerio de Salud Pública, 2012)

11. Política, Modelo y Plan Nacional Telemedicina/Telesalud: tiene como finalidad fortalecer el modelo de atención en salud relacionando el primer nivel de atención con los niveles superiores: secundario hospitales básicos-como terciario -hospitales especializados-. (Ministerio de Salud Pública del Ecuador/Proceso de Ciencia y Tecnología en Salud, 2010)

Debido a la interacción de varios elementos no se precisan otras normativas que sin duda tienen relación con las TIC, como la Ley n. ${ }^{\circ} 7$ de Derechos y Amparo del Paciente, la Ley Orgánica de la Defensoría del Pueblo, la Ley Orgánica de Defensa del Consumidor que proporcionan el marco legal a los proveedores del servicio de internet, y la Ley de Propiedad Intelectual entre las más importantes. 


\section{VENTAJAS Y LIMITACIONES DEL MARCO LEGAL}

La Constitución del Ecuador 2008 es garantista de derechos, constituye un cambio fundamental en el tema tratado y sin duda un avance para alcanzar la justicia y la equidad. El buen vivir visualiza al ser humano el centro estableciendo una relación diferente con el Estado, el mercado y la naturaleza. La salud, el acceso a las TIC, la propiedad de los datos personales y seguridad son derechos fundamentales garantizados por Estado, que al ser considerados como tales constituyen mandatos constitucionales que obligan a la implementación de políticas públicas, cuyos lineamientos se establecen en el PNBV. Asimismo, la ciencia y la tecnología tienen como finalidad la realización del Buen Vivir superando la visión de desarrollo supeditado al mercado. Por otro lado la recuperación de la rectoría proporciona el marco para el cambio institucional que debería regir en este campo configurando un escenario favorable para el desarrollo de las TIC y su aplicación en el ámbito de la salud.

La mayor jerarquía de la norma constitucional supone una obligación y proporciona el sustento jurídico sobre el cual se desarrollan leyes, reglamentos, normas, y demás cuerpos legales que actúan en el intersticio de la informática, la comunicación, la tecnología y la salud.
La Ley de Comercio Electrónico promueve el uso de la firma electrónica que norma el uso en la administración pública, es decir tiene relevancia en salud, aunque su origen está en el ámbito comercial.

En el caso de la Ley Especial de Telecomunicaciones y su reglamento con las modificaciones respectivas, mencionado con anterioridad, establecen la normativa general en el territorio nacional de aspectos relacionados con la comunicación. No obstante, su expedición se realizó antes de la vigencia de la Constitución.

La Ley Orgánica de Salud fomenta la ciencia y tecnología, constituyendo el marco general vigente que asociado a la LOSNS se refiere específicamente a las TIC y favorece su aplicación en la salud. Cabe resaltar que también estas leyes son previas a la norma constitucional vigente.

La ley del Sistema Nacional de Registro de Datos Públicos abarca los distintos momentos de atención a los pacientes o la gestión de los servicios que deben ser tratados con la debida confidencialidad y privacidad. El proceso de garantizar la protección de la información y los sistemas informáticos no es una actividad exclusivamente referida al 
campo de lo técnico; no en vano el respeto de la privacidad del paciente es un vínculo de confianza que se instaura entre él y la persona que lo atiende e involucra prácticas y actitudes referidas al uso de las tecnologías asociadas. (Torres \& Petrizzo, 2015)

Si bien la legislación vigente asegura la confidencialidad y privacidad de la información en salud, solo unos pocos países disponen de legislación específica que aborde la privacidad en la medicina aplicable en todos los ámbitos de la atención sanitaria, tal como se practica hoy en día, ya sea cara a cara o a través de las TIC. (Organización Mundial de la Salud, 2013)

Adicionalmente, es importante considerar que el MSP y el Ministerio de Telecomunicaciones, entre otros, son instancias sectoriales encargadas de la aplicación de las TIC en salud. EI MSP es la autoridad sanitaria nacional establecida tanto en la Constitución como en las Leyes, la diversidad de prestadores de servicios públicos y privados que si bien reconocen esta autoridad mantienen autonomía administrativa y financiera, lo cual implica que en muchos aspectos no hay la interoperabilidad entre los sistemas informáticos de los diferentes componentes del sistema de salud. El desarrollo de la Sociedad de la Información no puede reducirse a la adaptación de adelantos técnicos y a la creación de infraestructuras; si bien son cuestiones claves, los esfuerzos que se realicen en este sentido serán insuficientes sino se complementan con la educación, la cultura de la innovación en las empresas, los ajustes institucionales y regulatorios, y fundamentalmente, con el fomento de la investigación en TIC en la frontera de los avances actuales. (Ministerio de Telecomunicaciones y de la Sociedad de la Información, 2014)

\section{CONCLUSIONES Y RECOMENDACIONES}

La Constitución de 2008 ratifica el derecho a la salud y eleva a derecho el acceso a las TIC. Los mandatos legales obligan a implementar políticas públicas sobre asuntos determinados como de interés general y requiere un esfuerzo interinstitucional.

La ley o las normativas que tienen por objeto la regulación de materias in- formáticas y de comunicación en salud vigentes no obstaculizan las iniciativas que pueden desarrollar las instituciones para optimizar su aplicación. Sin embargo, requieren una actualización y articulación más detallada que permita compatibilizar los principios y garantías de la Constitución como sujetos de derechos, superando una visión de usuarios o clientes de servicios de salud o 
tecnologías y protegiendo la confidencialidad y seguridad de la información individual que garantice las libertades ciudadanas.

Los registros electrónicos se utilizan básicamente para actividades administrativas como registrar pacientes, citas, sistemas de gestión de medicamentos, personal, etc. Por ello, es necesario realizar un esfuerzo para la armonización técnica y normativa que facilite el intercambio de información al menos entre los servicios del MSP y la seguridad social pública.

Las voluntades por incorporar las TIC en todo tipo de aspectos en salud, aún incidiendo directamente en la calidad de servicios a los que accede la población, disminuyen brechas geográficas y económicas, no siendo una característica obligatoria en los servicios de salud tanto públicos como privados.

El uso de las TIC en salud enfrenta un prolijo marco legal. La autoridad y actores que intervienen en el proceso regulatorio son múltiples, producto de la complejidad del campo de acción, existiendo orientaciones en el ámbito internacional y regional. El reto está en favorecer la aplicación de las TIC, superando las restricciones legales y jurisdiccionales, que implica la interacción de la salud, la informática y las telecomunicaciones. 


\section{BIBLIOGRAFÍA}

Avella Martínez, L. Y. \& Parra Ruiz, P. (2013). Tecnologías de la Información y la Comunicación (TICS) en el Sector Salud. (Tesis de maestría, Universidad Nacional de Colombia). Recuperado de http://www.bdigital. unal .edu.co/11172/1/laurayanethavellamartinez. 2013.pdf

Barrera-Jaramillo, J. \& Gea-Izquierdo, E. (2013). La Tecnología NFC (Near Field Communication) en los servicios de salud. III Congreso Internacional Sobre Usos y Buenas prácticas con TIC. Málaga: Universidad de Málaga.

Carnicero, J. \& Fernández, A. (2012). Manual de Salud Electrónica para directivos de servicios y sistemas de salud. Santiago de Chile: Naciones Unidas.

Constitución de la República del Ecuador. (2008). Quito, Ecuador.

Decreto Ejecutivo 1790. (2001). Reglamento General a la Ley Especial de Telecomunicaciones Reformada- última modificación. Quito, Ecuador, 13 de octubre de 2011.

Decreto Ejecutivo 8. (2009). Registro Oficial n. ${ }^{\circ}$ 10. Quito, Ecuador, 24 de agosto de 2009.

Decisión 462. (1999). Normas que regulan el Proceso de Integración y Liberalización del Comercio de Servicios de Telecomunicaciones en la Comunidad Andina. Gaceta Oficial del Acuerdo de Cartagena n. 444. Cartagena, Colombia, 1 junio de 1999.

Eysenbach, G. (2001). What is e-health. Journal or Medicine Internet Research. Journal of Medical Internet. Recuperado de http:// www.jmir.org/2001/2/e20/doi10.2196/jmir. 3.2.e20

Hilbert, M., Bustos, S. \& Ferraz, J. C. (2005). Estrategias nacionales para la sociedad de la información en America Latina y el Caribe. CEPAL.

Indarte, S. \& Pazos Gutiérrez, P. (2011). Estándares e interoperabilidad en salud electrónica. Chile: CEPAL.

Jurado, R. (2006). Diagnóstico de las políticas de TIC en el Ecuador. Quito: FLACSO, Sede Ecuador.

Ley del Sistema Nacional de Registro de Datos Públicos, Ley s/n § Suplemento del Registro Oficial n. 162 (2010).

Ley n. 2002-67, Ley de Comercio Electrónico, Firmas Electrónicas y Mensajes de Datos § Registro Oficial n. ${ }^{\circ}$ 557-S (2002).

Ley Especial de Telecomunicaciones Reformada § Registro Oficial n. 992 (1992).

Ley n. ${ }^{\circ}$ 67, Ley Orgánica de Salud § Registro Oficial Suplemento n. ${ }^{\circ} 423$ (2006).

Ley n. ${ }^{\circ}$ 2002-80, Ley Orgánica del Sistema Nacional de Salud (2002).

Ministerio de Salud Pública. (2012). Estatuto Orgánico Sustitutivo de Gestión Organizacional por Procesos del MSP. Acuerdo Ministerial Registro Oficial n. 279.

Ministerio de Salud Pública del Ecuador/Proceso de Ciencia y Tecnología en Salud. (2010). Política, Modelo y Plan Nacional de Telemedicina/Telesalud. 
Ministerio de Telecomunicaciones y de la Sociedad de la Información. (2014). Tecnologías de la Información y Comunicaciones para el Desarrollo. Recuperado de http:// www.telecomunicaciones.gob.ec/wp-content/uploads/2015/09

Organización Mundial de la Salud. (2003). Informe de la Salud en el Mundo 2003. Forjemos el Futuro. Ginebra.

Organización Mundial de la Salud. (2013). Regímenes jurídicos de la cibersalud: informe basado en las conclusiones de la segunda encuesta mundial sobre Cibersalud. Ginebra.

Organización Panamericana de la Salud/Organización Mundial de la Salud. (2011). 51. ${ }^{\circ}$ Consejo Directivo. Estrategiay Plan de Acción sobre eSalud. Washington DC, EUA.

Rabanales Sotos, J., Párraga Martínez, I., Lopez-Torres, J., Prétel, F. \& Navarro Bravo, B. (2011). Tecnologías de la Información y Comunicaciones:Telemedicina. Revista Clínica de Medicina Familiar, 31 (5), 42-48.

Ramos, V. (2006). Las Tic en el sector de la salud. Research gate. Recuperado de https://www.researchgate.net/publication/2 168792_Las_TIC_en_el_sector_de_la_salud

Resolución 1415. (2011). Manual para la Producción Estadística de Tecnologías de la Información y Comunicación de la Comunidad Andina. Gaceta Oficial del Acuerdo de Cartagena n. ${ }^{\circ} 1951$.

Reglamento a la prestación de servicios de valor agregado. (2002). Resolución 071-03 CONATEL.
Sainz de Abajo, B., Rodrigues, J., García Salcines, E., Burón Fernandez, J., López Coronado, M. \& de Castro Lozano, C. (2011). M-Health y T-Health. La Evolución Natural del E-Health. Revista eSalud. Recuperado de dialnet-MHealthYTHealthLaEvolucionNaturalDeIEHealth-3407842.pdf

Secretaría Nacional de Planificación y Desarrollo. (2013). Plan Nacional para el Buen Vivir 2013-2017.

Torres Velandia, S. \& García Ponce de León, O. (2008). Redes de investigación y universidad pública. Revista Digital Universitaria, 9(2). Recuperado de http://www.revista. unam.mx/vol.9/num2/art06/int06.htm

Torres, J. \& Petrizzo, M. (2015). Sofware: programas libres y de código abierto en la Administración Pública. En D. Vila-Viñas, \& X. Barandiaran (Edits.), Buen Conocer/Flok Society Modelos sostenibles y políticas públicas para una economía social del conocimiento común y abierto en el Ecuador. Quito: IAEN-CIESPAL.

Universidad Técnica Particular de Loja. (2012). Manual de procesos y protocolos del proyecto "Telesalud UTPL Tutupaly". Recuperado de http://esalud.utpl.edu.ec/sites/default/files/publicaciones/manual_telesalud_r ural.pdf

Vance, C. (2015). Historia clínica electrónica se extiende por el país. Redacción Médica. Recuperado de http://www.redaccion medica.ec/noticia/medicos-tienen-historiaclinica-electronica-8663 